\title{
Le bassin versant expérimental urbain du Marais à Paris : origine et caractéristiques de la pollution des eaux pluviales urbaines en réseau d'assainissement unitaire
}

\author{
par M.-C. Gromaire-Mertz, G. Chebbo, M. Saad, J.-M. Mouchel \\ CERGRENE, 6-8 av. Blaise Pascal \\ 77455 Marne-la-Vallée Cedex 2
}

\section{I $\square$ INTRODUCTION}

De nombreuses études, s'appuyant souvent sur des campagnes expérimentales assez lourdes, ont été menées depuis les années 1970 [1] afin d'estimer les flux véhiculés par les réseaux d'assainissement séparatifs et unitaires, et de quantifier l'impact des rejets urbains de temps de pluie (RUTP) sur le milieu récepteur. Les résultats obtenus ont souligné l'importance de la pollution des RUTP, ils ont également fourni des éléments sur les caractéristiques de cette pollution, et des idées encore vagues et sûrement entachées d'erreurs sur la contribution des différentes sources à cette pollution. Cependant les données sur les caractéristiques de la pollution des eaux de ruissellement et leur contribution à la pollution des RUTP restent lacunaires, disparates et parfois contradictoires $[2,3,4]$.

Par ailleurs, les recherches récentes [3] montrent une aggravation de la pollution des RUTP en réseau d'assainis- sement unitaire, par rapport au réseau strictement pluvial. Les surverses unitaires sont globalement plus chargées en matière organique que les rejets strictement pluviaux. De plus, les matières en suspension dans les rejets unitaires ont des vitesses de chute inférieures à celles des rejets strictement pluviaux, ce qui rend leur traitement plus difficile. Peu d'informations sont disponibles à l'heure actuelle sur les facteurs expliquant ces différences entre réseaux séparatifs et unitaires. Il semblerait que la seule présence des eaux usées ne suffise pas à expliquer cette aggravation et c'est vers la remise en suspension des dépôts formés dans le réseau unitaire que s'orientent les soupçons [3,4].

Dans ce contexte, le CERGRENE a lancé en 1994, un programme de recherche intitulé "Génération et Transport de la Pollution des Rejets Urbains de Temps de Pluie en Réseau d'Assainissement Unitaire ». Cet article a pour objet de décrire le programme de recherche qui est mené actuellement sur le bassin versant expérimental du Marais et de présenter les premiers résultats.

An experimental urban catchment has been created in the centre of Paris, in order to obtain a description of the pollution of urban wet weather flows at different levels of the combined sewer system, and to estimate the contribution of runoff, waste water and sewer sediments to this pollution. Thirty-six rainfall events were studied from May to December 1996. Dry weather flow was monitered for one week. Roof, street and yard runoff, total flow at the catchment outlet and waste water were analysed for SS, VSS, COD and $B O D 5$, on both total and dissolved fraction. Results show an evolution in the characteristics of wet weather flow from up to dowstream: concentrations increase from the catchment entry to the outlet, as well as the proportion of particle-bound pollutants and the part of organic matter. A first evaluation of the different sources of pollution establishes that a major part of wet weather flow pollution originates from inside the combined sewer, probably through erosion of sewer sediments. 


\section{II 口 LE PROGRAMME DE RECHERCHE}

\subsection{Objectifs}

Le programme de recherche «Génération et transport de la pollution des rejets urbains par temps de pluie " vise à équiper un bassin versant urbain, situé en centre ville et drainé par un réseau unitaire, d'un dispositif expérimental permettant d'étudier la pollution des eaux pluviales aux différents niveaux du cycle de l'eau dans la ville. Il poursuit un triple objectif :

- évaluer, sur un même site, la contribution des différentes sources à la pollution des eaux de temps de pluie,

- mieux caractériser la pollution issue de chaque source,

- étudier les mécanismes de formation et de transfert de cette pollution en surface et dans le réseau d'assainissement unitaire.

Ce type de recherche devrait permettre d'orienter des solutions de traitement de la pollution des eaux de temps de pluie, tant curatives que préventives et fournir les bases pour la conception de modèles de simulation des flux plus précis que ceux disponibles actuellement.

\subsection{Méthodologie}

Nous avons distingué quatre sources à la pollution des rejets pluviaux urbains en réseau unitaire :

- les retombées atmosphériques par voie sèche et humide,

- le lessivage des chaussées, toitures et cours intérieures,

- les eaux usées,

- les stocks constitués dans le réseau d'assainissement.

Les eaux de ruissellement sont caractérisées et quantifiées au niveau de sept tronçons de rue, quatre toitures et trois cours du bassin versant expérimental (Figure 1). L'étude des retombées atmosphériques sèches et humides permet d'évaluer la contribution des apports atmosphériques en MES, métaux et hydrocarbures à la pollution de ces eaux de ruissellement. Par ailleurs il est prévu d'étudier les mécanismes d'accumulation et d'entraînement des stocks de pollution sur les chaussées.

La pollution des eaux usées est caractérisée et quantifiée par temps sec à l'exutoire du bassin versant.

Il est très difficile d'évaluer et de caractériser directement la contribution des stocks en réseau à la pollution des rejets urbains de temps de pluie. Aussi avons-nous recours à différentes méthodes indirectes dont nous confronterons les résultats :

- établissement de bilans de masse, à l'échelle de l'événement pluvial, entre les apports provenant des eaux de ruissellement et des eaux usées, et la pollution totale mesurée à l'exutoire du bassin versant,

- traçage (comparaison des caractéristiques physico-chimiques de l'effluent entre l'entrée et la sortie du réseau),

- suivis de stocks de dépôts en réseau.

Par ailleurs une étude spécifique des dépôts va être menée dans le réseau d'assainissement du bassin expérimental du Marais. Elle vise à identifier les différents types de dépôts existants, à évaluer leur contribution relative à la pollution des RUTP et à étudier leurs mécanismes de formation et d'entraînement. La méthodologie suivie repose sur une typologie des dépôts (caractéristiques, conditions d'existence et potentiel polluant) et sur un suivi approfondi de l'évolution par temps sec et par temps de pluie de quelques gisements de dépôts.

\subsection{Paramètres mesurés}

L'étude porte sur les paramètres de pollution suivants : matières en suspension, matières volatiles en suspension, demande chimique en oxygène, demande biochimique en oxygène, hydrocarbures, métaux lourds (cadmium, cuivre, plomb, zinc). Ces analyses sont réalisées sur les fractions totales et dissoutes (après filtration à $0,45 \mu \mathrm{m}$ ), ainsi qu'après fractionnement des solides en suspension par classes de vitesse de chute. Les composés organiques volatils (COV) sont également analysés. De plus, nous comptons analyser la mobilité des métaux par spéciation géochimique, ainsi que chaque composé des familles d'hydrocarbures aromatiques et aliphatiques.

\subsection{Equipes de recherche et financement}

Cette recherche, pilotée par le CERGRENE, est aujourd'hui réalisée en collaboration entre le CERGRENE, le LABAM et l'IHC. Le tableau 1 synthétise les différents thèmes de recherches abordés, en précisant les organismes financeurs.

Tableau 1. - Thèmes de recherche et organismes financeurs.

\begin{tabular}{|c|c|c|}
\hline \multicolumn{2}{|l|}{ Thème de recherche } & Financement \\
\hline \multicolumn{2}{|c|}{$\begin{array}{l}\text { Retombées atmosphériques sèches et humides } \\
\text { de MES, métaux, hydrocarbures et COV }\end{array}$} & \multirow{3}{*}{$\begin{array}{l}\text { PIREN - SEINE } \\
\text { Groupe Bassins Versants Urbains }\end{array}$} \\
\hline \multicolumn{2}{|c|}{$\begin{array}{l}\text { Mécanismes d'accumulation et d'entraînement } \\
\text { des stocks sur les chaussées }\end{array}$} & \\
\hline $\begin{array}{l}\text { Caractéristiques et flux } \\
\text { des eaux de ruissellement } \\
\text { de surface }\end{array}$ & $\begin{array}{l}\text { spéciation des métaux, } \\
\text { analyse des } \\
\text { différents composés } \\
\text { d'hydrocarbures, COV }\end{array}$ & \\
\hline $\begin{array}{l}\text { Caractéristiques et flux } \\
\text { des eaux de temps sec } \\
\text { Caractéristiques et flux } \\
\text { des eaux de temps de } \\
\text { pluie à l'exutoire }\end{array}$ & $\begin{array}{l}\text { concentration totale et } \\
\text { dissoute en : MES, MVS, } \\
\text { DCO, DBO5, métaux, hydrocarbures, } \\
\text { répartition par classes de vitesses de chute, } \\
\text { répartition au cours de l'événement } \\
\text { pluvieux }\end{array}$ & \multirow{2}{*}{$\begin{array}{l}\text { Comité Inter Agences de l'Eau } \\
\text { Ville de Paris } \\
\text { Conseil régional d'lle de France } \\
\text { Conseil Scientifique et Technique } \\
\text { des Bassins Versants de } \\
\text { Recherche Expérimentaux } \\
\text { Laboratoire Central des Ponts et Chaussées } \\
\text { Ecole Nationale des Ponts et Chaussées }\end{array}$} \\
\hline \multicolumn{2}{|c|}{$\begin{array}{l}\text { Mécanismes d'accumulation et } \\
\text { d'entraînement de la pollution en réseau }\end{array}$} & \\
\hline
\end{tabular}




\section{LE BASSIN VERSANT DU MARAIS}

Le bassin versant expérimental du Marais est situé au centre de Paris, sur une partie des $3^{\mathrm{e}}$ et $4^{\mathrm{e}}$ arrondissements. Sa surface est de 42 hectares, ce qui permet une connaissance détaillée du site. L'occupation du sol est représentative d'un centre ville ancien : il s'agit d'un quartier résidentiel avec de nombreux petits commerces et des activités du secteur tertiaire mais peu d'activités industrielles. La répartition des surfaces est la suivante : toitures $54,5 \%$, chaussées $22,5 \%$, squares, cours et jardins $23 \%$. Le coefficient de ruissellement est d'environ 0,78 et la pente moyenne du bassin est de $0,84 \%$. L'habitat est dense, avec une population totale de 12400 habitants.

Ce bassin versant possède un réseau d'assainissement unitaire, ramifié et entièrement visitable, muni d'avaloirs non sélectifs pour le drainage des chaussées. Il comprend trois collecteurs à banquettes, d'une longueur totale de $1,8 \mathrm{~km}$, drainant une cinquantaine d'égouts élémentaires, d'une longueur totale de $5,8 \mathrm{~km}$. La pente moyenne des égouts est de $0,8 \%$ alors que celle des collecteurs est inférieure à $0,1 \%$. Le bassin versant est bien délimité et isolé des bassins alentour.

Un nettoyage régulier est pratiqué sur l'ensemble de la voirie du bassin versant. Les caniveaux sont balayés quotidiennement. Trottoirs et caniveaux sont nettoyés avec un jet d'eau sous pression deux à cinq fois par semaine. Des aspiratrices de chaussée circulent quotidiennement, du lundi au vendredi, sur la plupart des rues du bassin.

\section{IV — LES ÉQUIPEMENTS}

Les équipements actuellement installés sur le bassin versant permettent la caractérisation et la quantification de la pollution des eaux pluviales de l'atmosphère jusqu'à l'exutoire du bassin, ainsi que l'étude des eaux de temps sec. Pour le cas particulier des composés organiques volatils, les échantillons sont prélevés manuellement dans des flacons sertis (phase liquide) ou des seringues étanches (phase gazeuse).

\subsection{Retombées atmosphériques et pluviométrie}

Les retombées atmosphériques sèches et humides sont échantillonnées au moyen d'un appareil "ARS 1000 " (MTX-Italia, Italie). Cet échantillonneur automatique sur pied $(90 \mathrm{~cm})$ est composé de deux réservoirs en polyéthylène ayant une surface ouverte de $660 \mathrm{~cm}^{2}$, et d'un couvercle mobile dont la position est commandée électroniquement par l'intermédiaire d'un détecteur de précipitation. La retombée humide est récoltée dans un réceptacle de 17 litres. La retombée sèche est échantillonnée dans un film de $1 \mathrm{~cm}$ d'eau osmosée-permutée à $18 \mathrm{M} \Omega$ (hauteur maintenue constante pendant toute la période d'échantillonnage à l'aide d'une pompe). L'appareil est placé sur une toiture terrasse du bassin ( $4^{\mathrm{C}}$ étage). Un relevé hebdomadaire des échantillons est effectué.

Le bassin versant est équipé de deux pluviographes à augets basculeurs, placés sur des toitures terrasses. On enregistre l'heure de chaque basculement.

\subsection{Ruissellement des toitures, des cours et des chaus- sées}

Des échantillons moyens des eaux de ruissellement sont collectés au niveau des descentes de gouttières de quatre toits du bassin. Ces toits sont représentatifs des différents types de couvertures utilisés sur le bassin versant : tuile mécanique, tuile plate, tôle de zinc et ardoise. Les échantillons sont prélevés par piquage dans la gouttière et collectés dans des fûts de 100 litres.

Trois cours intérieures du bassin versant ont été équipées d'un préleveur automatique permettant d'échantillonner les eaux de ruissellement. Ces cours, de caractéristiques très différentes, sont représentatives des types de cours du bassin : pavée, bétonnée et arborée, gazon et gravier. Les préleveurs sont déclenchés par un détecteur de pluie. L'échantillonnage se fait à pas de temps constant dans la bouche de drainage de la cour.

Le choix du nombre de sites d'étude des eaux de ruissellement de chaussée et leur emplacement est basé sur une analyse statistique des caractéristiques des rues. Suite à une

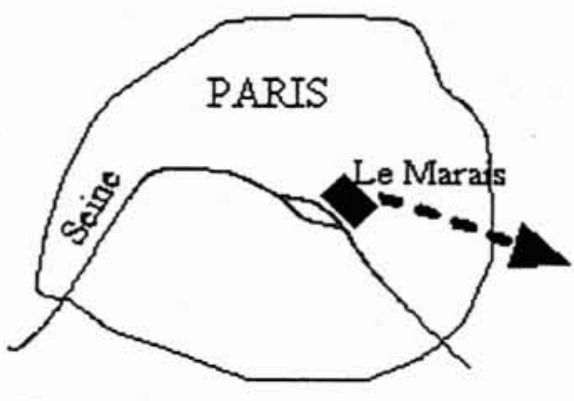

E: exutoire du bassin

A: avaloirs équiqés

T: toitures équipées

C: cours équịées

P: pluviographes

$\mathrm{R}$ : préleveur de retombées atmosphériques

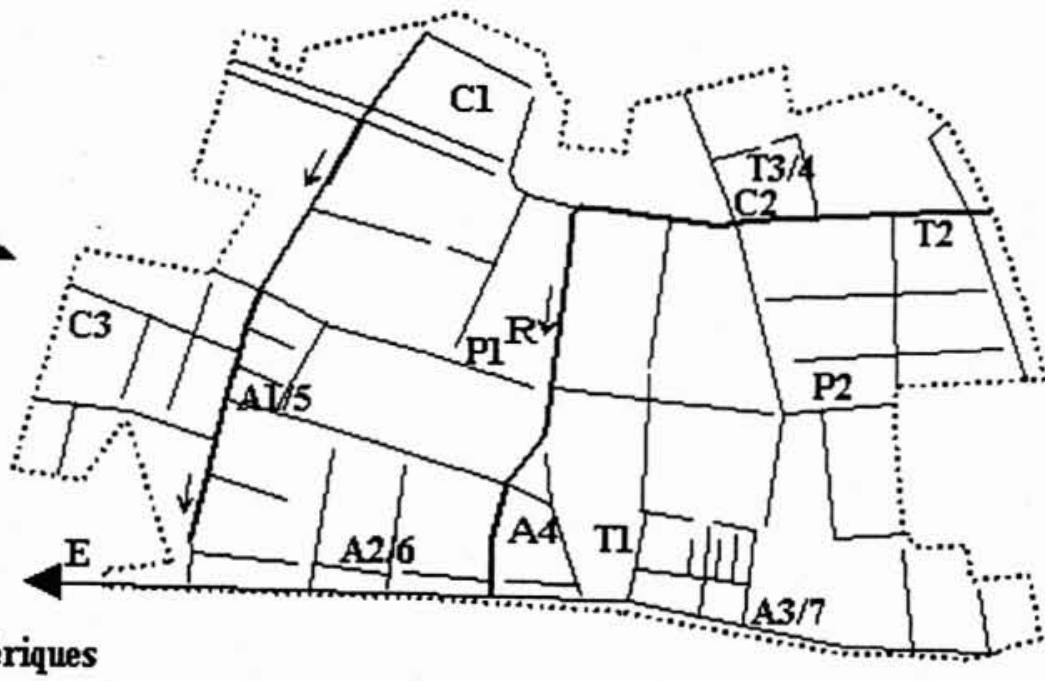

1. Plan du bassin versant et localisation des points de mesure. 


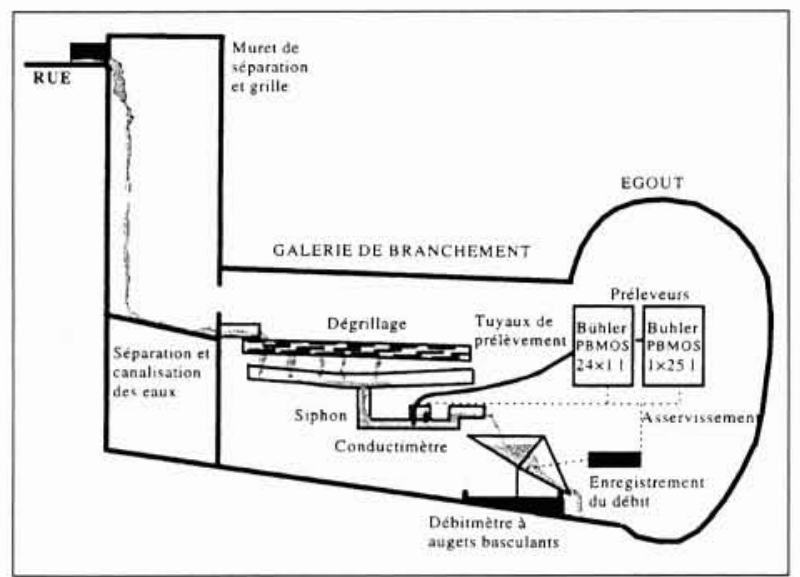

2. Equipement d'un avaloir pour l'étude des eaux de ruissellement de chaussée.

enquête menée sur l'ensemble des rues du bassin et portant sur les paramètres susceptibles d'influencer les phénomènes d'accumulation et d'entraînement des polluants (trafic routier, fréquentation piétonne, activité commerciale, pente, type de revêtement, usure, ...) nous avons pu identifier sept groupes de rues relativement homogènes. Quatre avaloirs situés à des coins de rues ont été choisis. Chacun de ces avaloirs draine deux tronçons de rues appartenant à deux groupes différents, avec au total huit tronçons de rues appartenant aux sept groupes différents.

L'équipement des avaloirs (figure 2) a consisté à séparer les eaux drainées de part et d'autre de l'avaloir par un mur, les canaliser, mesurer leur débit et prélever des échantillons. Suivant la surface de la chaussée drainée, le débit est mesuré avec des augets basculants de 20 litres ou avec un déversoir triangulaire. Chaque avaloir est équipé de deux préleveurs automatiques Bühler-PBMOS (un préleveur multiflacon pour l'établissement de pollutogrammes, un préleveur monoflacon pour la mesure des vitesses de chute) asservis aux débitmètres. L'échantillonnage est déclenché lorsqu'un écoulement de conductivité inférieure à $450 \mu \mathrm{S} / \mathrm{cm}$ est détecté dans l'avaloir, pour éviter de prélever les eaux de lavage. La fréquence de prélèvement est proportionnelle au volume écoulé. Pour des raisons techniques et financières, nous nous limitons à l'étude de trois tronçons de rues par événement pluvieux.

\subsection{Exutoire (fig. 3)}

Les débits à l'exutoire du bassin versant sont mesurés avec un appareil Ultraflux, par mesure simultanée de la hauteur d'eau et de la vitesse, et enregistrés à un pas de deux minutes. La vitesse moyenne dans l'écoulement est calculée à partir du temps de transit des ondes ultrasonores sur quatre cordes. Une double mesure de la hauteur d'eau est réalisée avec un ultrason aérien et un capteur de pression piézométrique.

Ce débitmètre asservit deux préleveurs automatiques. L'un muni de 24 flacons de 2,9 litres (Bühler PP92) sert à l'établissement de pollutogrammes; l'autre muni d'un monoflacon de 70 litres (Bühler Vegamon 94) fournit un échantillon moyen pour la mesure de la vitesse de chute, la spéciation des métaux et l'identification des différents composés d'hydrocarbures. Les prélèvements sont déclenchés lorsque le niveau d'eau dépasse le niveau maximal de temps sec. La fréquence de prélèvement est proportionnelle au volume écoulé dans le collecteur.

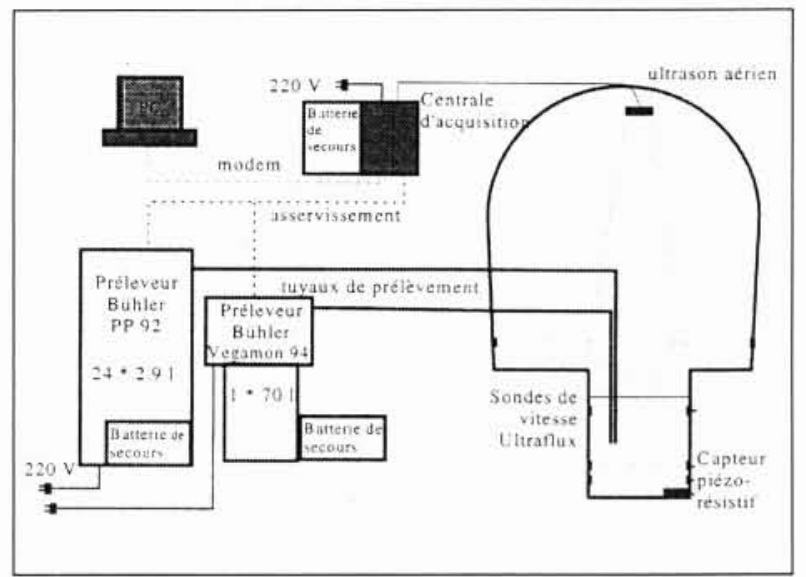

3. Equipement de l'exutoire.

\section{DISPONIBILITÉ DU BASSIN VER- SANT}

Les équipements du bassin versant mis en place pour l'étude des eaux pluviales sont opérationnels depuis juin 1996 et la campagne de mesure actuelle devrait se poursuivre pendant au moins un an. Par ailleurs, les techniques et les appareillages nécessaires à l'étude des mécanismes d'accumulation et d'entraînement des stocks de pollution en surface et en réseau sont en cours de développement.

\section{VI —ÉTUDE DES EAUX DE TEMPS SEC}

Les eaux de temps sec à l'exutoire du bassin versant ont été caractérisées pour six différents jours de la semaine, pendant la période du 15 au 27 juillet 1996. Chaque jour, les concentrations en MES, MVS, DCO et DBO5 ont été mesurées sur 24 échantillons moyens horaires. Par ailleurs les débits sont enregistrés en continu depuis le début de l'étude à un pas de temps de 2 minutes.

Le débit moyen à l'exutoire du bassin versant est de 565 litres par jour et par habitant. Ce débit élevé comparé au chiffre de 100 à 300 l/hab/jour cité par Saget et Chebbo [5], peut s'expliquer par l'importance des eaux de lavage de voirie, les activités professionnelles (restauration, commerces et bureaux) et par un apport d'eaux parasites. Comme le montre le tableau 2, les charges moyennes des eaux usées mesurées sur le bassin versant expérimental sont comparables à celles généralement observées en France.

On observe clairement un cycle journalier des débits et des concentrations : les valeurs maximales sont mesurées entre 10 et 11 heures, avec un second pic plus faible en début d'après midi, les valeurs minimales sont atteintes entre 5 et 6 h du matin (fig. 4). Ce cycle journalier est stable, avec de faibles différences entre jours de semaine et week-ends. Les concentrations varient en phase avec le débit ; cependant leur plage de variation est largement supérieure à celle du débit : la variation d'un facteur 3 des débits $(0,047$ à $0,124 \mathrm{~m}^{3} / \mathrm{s}$ ) s'accompagne d'une variation d'un facteur 10 des concentrations en MES ( 23 à $225 \mathrm{mg} / \mathrm{l})$. Cela peut s'expliquer en partie par la présence d'eaux parasites qui induisent une dilution des effluents d'autant plus importante que les débits d'eaux usées sont faibles. Le phénomène peut également être lié à une érosion des dépôts pendant le pic de débit diurne et une sédimentation la nuit lorsque l'énergie de 
Tableau 2.- Charges moyennes des eaux usées.

\begin{tabular}{|c|c|c|c|c|c|c|c|}
\hline & \multicolumn{2}{|c|}{ MES } & \multicolumn{2}{|c|}{ DBO5 } & \multicolumn{2}{|c|}{ DCO } & \multirow{2}{*}{$\frac{\text { MVS/MES }}{\%}$} \\
\hline & g/jour/hab. & $\mathrm{mg} / \mathrm{l}$ & g/jour/hab. & $\mathrm{mg} / \mathrm{l}$ & DCO/DBO5 & $\mathrm{mg} / \mathrm{l}$ & \\
\hline Paris-Le Marais & 70 & 125 & 84 & 150 & 1,94 & 290 & 83 \\
\hline $\begin{array}{c}\text { Degrémont } \\
\text { (1989), Chebbo } \\
\text { (1992), Chebbo et al. (1995) }\end{array}$ & 70 à 90 & 100 à 500 & 60 à 80 & 100 à 500 & 2 à 2,5 & 200 à 1000 & 65 à 85 \\
\hline
\end{tabular}

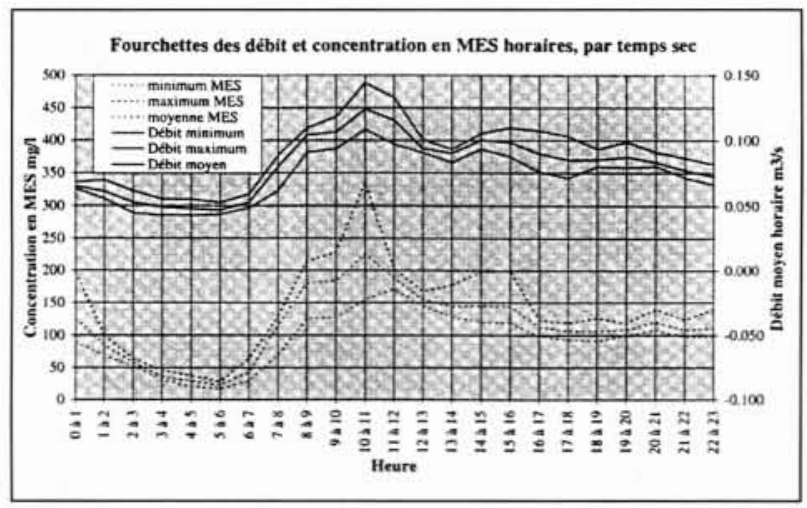

4. Variations journalières des débits et des concentrations en MES.

l'écoulement est faible. Par ailleurs, des variations de qualité des eaux usées au cours de la journée, liées en particulier aux activités professionnelles, ne sont pas exclues.

\section{VII —CARACTÉRISTIQUES DES ÉVÉNE- MENTS PLUVIEUX ÉUDIÉS}

Nous avons défini comme événement pluvieux une précipitation d'une hauteur totale supérieure à $1 \mathrm{~mm}$, et séparée de l'événement précédent par une durée de temps sec d'au moins 30 minutes (temps de concentration du bassin). La campagne de mesure a porté sur 36 événements pluvieux, de caractéristiques variées, entre mai et décembre 1996 (tabl. 3).

Cependant, les mesures n'ont pas été effectuées sur l'ensemble des sites pour toutes les pluies. On trouvera en tableau 4 le nombre de pluies étudiées pour chaque site de mesure.

\section{VIII —CONCENTRATIONS MOYENNES PAR ÉVÉNEMENT PLUVIEUX}

Pour les eaux de ruissellement, on mesure des concentrations moyennes en DBO5 faibles. Les concentrations moyennes en MES et en DCO ainsi que les taux de matières volatiles sont extrêmement variables d'une pluie à une autre.

Les concentrations dans les eaux de toitures sont généralement faibles. Cependant, des valeurs allant jusqu'à 210 $\mathrm{mg} / \mathrm{l}$ pour les MES et $198 \mathrm{mg} / \mathrm{l}$ pour la DCO ont été mesurées lors de certaines pluies. Ces concentrations s'avèren liées à la durée de temps sec, l'intensité moyenne, l'intensité maximale et la durée totale de la pluie. Nous n'avons pas observé de variations significatives des MES, MVS, DCO et DBO5 d'un type de toiture à un autre.

En ce qui concerne les eaux de ruissellement des cours intérieures et des chaussées, les résultats dépendent fortement de l'occupation du sol et diffèrent d'un site à un autre. L'arrachement de terre dans la cour 3 (gravier) conduit à des valeurs de MES très fortes, avec un taux de MVS faible. Les différences entre les cours 1 et 2 s'expliquent par les pratiques de nettoyage (lavage régulier pour la cour 2 , aucun

Tableau 3.- Caractéristiques des événements pluvieux.

\begin{tabular}{|c|c|c|c|c|c|}
\cline { 2 - 6 } \multicolumn{1}{c|}{} & Hauteur d'eau $(\mathrm{mm})$ & Imoy $(\mathrm{mm} / \mathrm{h})$ & $\operatorname{Imax}(\mathrm{mm} / \mathrm{h})$ & Durée $(\mathrm{h}: \mathrm{mn})$ & Temps sec $(\mathrm{j})$ \\
\hline minimum & 1 & 1,4 & 1,2 & $00: 10$ & 0,03 \\
\hline maximum & 14,6 & 42 & 180 & $07: 27$ & 29,91 \\
\hline médiane & 4 & 5 & 8 & $01: 40$ & 1 \\
\hline
\end{tabular}

Tableau 4. - Nombre d'événements pluvieux par site.

\begin{tabular}{|c|c|c|c|c|c|c|c|c|c|c|c|}
\hline \multicolumn{4}{|c|}{ Toitures } & \multicolumn{3}{c|}{ Cours intérieures } & \multicolumn{3}{c|}{ Chaussées } & Exutoire \\
\hline Tuile mécanique & Tuile plate & Zinc & Ardoise & Béton & Pavés & Gravier et gazon & Rue 1 & Rue 2 & Rue 3 & Rue 4 & \\
\hline 29 & 33 & 33 & 33 & 12 & 11 & 5 & 21 & 18 & 18 & 10 & 34 \\
\hline
\end{tabular}




\begin{tabular}{|c|c|c|c|c|c|c|c|c|c|c|c|c|c|}
\hline & & \multicolumn{3}{|c|}{ MES mg/l } & \multicolumn{3}{|c|}{$\mathrm{DCO} \mathrm{mg} / \mathrm{l}$} & \multicolumn{3}{|c|}{ DBO5 mg/l } & \multicolumn{3}{|c|}{$\%$ MVS/MES } \\
\hline & & $\min$ & $\max$ & moy & $\min$ & $\max$ & moy & $\min$ & $\max$ & moy & $\min$ & $\max$ & moy \\
\hline Toitures & $\begin{array}{c}\text { tuile brune } \\
\text { tuile rouge } \\
\text { zinc } \\
\text { ardoise }\end{array}$ & $\begin{array}{l}4 \\
4 \\
5 \\
5\end{array}$ & $\begin{array}{c}211 \\
84 \\
131 \\
91\end{array}$ & $\begin{array}{l}39 \\
27 \\
31 \\
20\end{array}$ & $\begin{array}{l}9 \\
7 \\
9 \\
5\end{array}$ & $\begin{array}{c}120 \\
91 \\
156 \\
198\end{array}$ & $\begin{array}{l}28 \\
32 \\
35 \\
26\end{array}$ & $\begin{array}{l}2,4 \\
1,5 \\
1,5 \\
1,6\end{array}$ & $\begin{array}{l}13 \\
11 \\
15 \\
42\end{array}$ & $\begin{array}{l}5 \\
5 \\
5 \\
5\end{array}$ & $\begin{array}{l}14 \\
32 \\
33 \\
28\end{array}$ & $\begin{array}{l}66 \\
67 \\
72 \\
62\end{array}$ & $\begin{array}{l}35 \\
48 \\
45 \\
43\end{array}$ \\
\hline Cours & $\begin{array}{c}1 \text { (béton) } \\
2 \text { (pavés) } \\
3 \text { (graviers) }\end{array}$ & $\begin{array}{c}22 \\
9 \\
32\end{array}$ & $\begin{array}{c}109 \\
38 \\
490\end{array}$ & $\begin{array}{c}55 \\
18 \\
201\end{array}$ & $\begin{array}{l}59 \\
17 \\
42\end{array}$ & $\begin{array}{l}263 \\
71 \\
211\end{array}$ & $\begin{array}{l}134 \\
37 \\
89\end{array}$ & $\begin{array}{l}13 \\
6 \\
8\end{array}$ & $\begin{array}{l}47 \\
16 \\
27\end{array}$ & $\begin{array}{l}23 \\
10 \\
18\end{array}$ & $\begin{array}{l}60 \\
44 \\
12\end{array}$ & $\begin{array}{l}77 \\
71 \\
27\end{array}$ & $\begin{array}{l}70 \\
64 \\
17\end{array}$ \\
\hline Chaussées & $\begin{array}{l}\text { rue } 1 \\
\text { rue } 2 \\
\text { rue } 3 \\
\text { rue } 4\end{array}$ & $\begin{array}{l}57 \\
41 \\
10 \\
22\end{array}$ & $\begin{array}{l}1006 \\
206 \\
457 \\
206\end{array}$ & $\begin{array}{c}278 \\
82 \\
156 \\
85\end{array}$ & $\begin{array}{l}124 \\
56 \\
25 \\
40\end{array}$ & $\begin{array}{l}1102 \\
271 \\
452 \\
344\end{array}$ & $\begin{array}{l}425 \\
119 \\
195 \\
150\end{array}$ & $\begin{array}{l}28 \\
16 \\
14 \\
10\end{array}$ & $\begin{array}{c}224 \\
46 \\
70 \\
74\end{array}$ & $\begin{array}{l}91 \\
28 \\
36 \\
35\end{array}$ & $\begin{array}{l}49 \\
41 \\
33 \\
54\end{array}$ & $\begin{array}{l}70 \\
71 \\
61 \\
70\end{array}$ & $\begin{array}{l}58 \\
58 \\
49 \\
62\end{array}$ \\
\hline Exutoire & Exutoire & 105 & 559 & 277 & 123 & 831 & 405 & 59 & 296 & 170 & 53 & 81 & 70 \\
\hline
\end{tabular}

5. Concentrations moyennes mesurées sur les diférents sites, pour les pluies étudiées.

entretien pour la cour 1) et la présence d'arbres dans la cour 1. Des concentrations particulièrement fortes ont été mesurées pour le ruissellement de la rue 1 (étroite mais très fréquentée, avec de nombreux snacks et une circulation automobile difficile) et dans une moindre mesure pour la rue 3 (avenue à forte circulation automobile et piétonne). Les eaux de ruissellement des rues 2 et 4 dont les caractéristiques sont voisines (peu fréquentées, pas de commerces) ont des charges polluantes semblables et bien inférieures à celles de la rue 1 .

Les concentrations mesurées à l'exutoire du bassin versant sont similaires à celles citées par Saget et Chebbo [5] pour différents réseaux unitaires en France.

Remarquons que pour la plupart des événements pluvieux les concentrations mesurées à l'exutoire du bassin versant étaient équivalentes ou supérieures à celles observées par temps sec à la même période de la journée. Les eaux de ruissellement étant en général bien moins concentrées, cela suggère une contribution venant des dépôts en réseau d'assainissement.

\section{DÉPARTITION DE LA POLLU- TION ENTRE FRACTION DISSOU- TE ET FRACTION PARTICULAIRE}

Le pourcentage de la pollution totale en DCO et DBO5 lié aux particules augmente de l'amont vers l'aval du bassin versant (tabl. 5). Par temps de pluie à l'exutoire du bassin versant, 70 à $90 \%$ de la pollution totale en DCO et en DBO5 est liée aux particules, ce qui confirme les résultats des études précédentes [3]. La contribution de la fraction dissoute est nettement plus importante dans le cas des eaux
Tableau 5. - Répartition de la pollution entre fractions dissoute et particulaire.

\begin{tabular}{|c|ccc|ccc|}
\cline { 2 - 6 } \multicolumn{1}{c|}{} & \multicolumn{3}{c|}{$\%$ DCO particulaire } & \multicolumn{3}{c|}{ DBO5 particulaire } \\
\cline { 2 - 7 } \multicolumn{1}{c|}{} & min & $\max$ & $\operatorname{moy}$ & $\min$ & $\max$ & $\operatorname{moy}$ \\
\hline TOITURES & 22 & 94 & 57 & 17 & 84 & 46 \\
\hline COURS & 35 & 86 & 61 & 28 & 82 & 56 \\
\hline AVALOIRS & 24 & 94 & 72 & 47 & 93 & 65 \\
\hline EXUTOIRE & 72 & 92 & 82 & 62 & 91 & 79 \\
\hline
\end{tabular}

de ruissellement. Les pourcentages de pollution dissoute les plus forts sont mesurés sur les eaux de toitures, avec cependant d'importantes fluctuations d'une pluie à une autre (17 à $94 \%$ ). Dans les eaux de temps sec, à l'exutoire du bassin, 65 à $85 \%$ de la DCO et 50 à $75 \%$ de la DBO5 sont liés aux particules.

\section{CONTRIBUTION DES DIFFÉREN- TES SOURCES}

On peut identifier trois sources à la pollution des RUTP : les eaux de temps sec, les eaux de ruissellement, les dépôts en réseau d'assainissement. Nous avons calculé les contributions relatives de chacune de ces sources pour cinq événements pluvieux (tabl. 6) de notre campagne de mesure, en appliquant la méthode suivante :

Tableau 6. - Contribution des différentes sources de pollution pour 5 événements pluvieux.

\begin{tabular}{|c|c|c|c|c|c|c|c|c|c|}
\hline \multicolumn{5}{|c|}{ Caractéristiques de l'événement pluvieux } & \multicolumn{5}{|c|}{$\%$ de contribution à la masse totale de MES } \\
\hline Date & Heure début & $\begin{array}{c}\text { Durée pluie } \\
\text { (mm) }\end{array}$ & Imoy $(\mathrm{mm} / \mathrm{h})$ & $\begin{array}{c}\text { Htot } \\
(\mathrm{mm})\end{array}$ & Eaux usées & Toitures & Cours & Avaloirs & Réseau \\
\hline 05/07/1996 & $04: 00$ & 280 & $+4,5$ & 21,6 & $28 \%$ & $6 \%$ & $5 \%$ & $15 \%$ & $47 \%$ \\
\hline $\begin{array}{l}10 / 08 / 1996 \\
11 / 08 / 1996 \\
12 / 08 / 1996 \\
19 / 09 / 1996\end{array}$ & $\begin{array}{l}17: 00 \\
16: 30 \\
03: 00 \\
09: 30\end{array}$ & $\begin{array}{c}180 \\
13 \\
45 \\
435\end{array}$ & $\begin{array}{c}4,7 \\
35,3 \\
5,6 \\
1,8\end{array}$ & $\begin{array}{c}14 \\
7,6 \\
4,2 \\
13,4\end{array}$ & $\begin{array}{r}9 \% \\
7 \% \\
6 \% \\
37 \%\end{array}$ & $\begin{array}{c}10 \% \\
10 \% \\
23 \% \\
3 \%\end{array}$ & $\begin{array}{r}4 \% \\
7 \% \\
10 \% \\
3 \%\end{array}$ & $\begin{array}{l}17 \% \\
10 \% \\
10 \% \\
11 \%\end{array}$ & $\begin{array}{l}59 \% \\
66 \% \\
50 \% \\
45 \%\end{array}$ \\
\hline
\end{tabular}


- masse de pollution provenant des eaux de ruissellement (MPRuis) :

- on affecte la moyenne des concentrations mesurées pour cet événement sur les différents échantillons de toitures (respectivement de cours et de chaussées) à la surface totale de toitures (respectivement de cours et de chaussées) du bassin versant ;

- le volume de ruissellement est calculé à partir d'un coefficient de ruissellement théorique et de la hauteur de la pluie; - masse de pollution des eaux usées (MPEU) : les concentrations et les volumes utilisés pour le calcul sont ceux mesurés par temps sec à l'exutoire du bassin versant à la même heure, le même jour de la semaine ;

- masse totale de pollution (MPTot) : elle est calculée à partir des mesures de débit et de concentration au cours de la pluie, à l'exutoire du bassin ;

- masse de pollution provenant des dépôts (MPD) : MPD = MPTot - MPEU - MPRuis.

Les résultats (tabl. 6) montrent une contribution très importante des dépôts à la pollution des RUTP. Pour ces cinq événements pluvieux, 40 à $60 \%$ des masses totales en MES et en DCO ainsi que 40 à $75 \%$ des masses totales en DBO5 et en MVS proviennent du réseau d'assainissement. Les eaux de ruissellement apportent environ $30 \%$ de la masse totale en MES et DCO, $20 \%$ de la masse de matières volatiles et moins de $20 \%$ de la DBO5. La contribution des eaux usées varie suivant l'événement pluvieux : négligeable la nuit et pour les événements cours et intenses, elle est significative pour des événements faibles et de longue durée. Cette contribution est plus importante pour la DBO5 et la DCO que pour les MES.

Ces résultats sont comparables à ceux obtenus par Krejci et al. [4] et Bachoc [8]. Krejci a calculé une contribution du réseau à $59 \%$ (dont $20 \%$ provenant des biofilms et $34 \%$ des dépôts) pour quatre événements pluvieux sur un petit bassin versant $(12,7$ ha) à Lausanne. Bachoc trouve une contribution des dépôts de 30 à $45 \%$ des MES, lors de trois pluies à l'exutoire du collecteur 13 à Marseille. A l'échelle annuelle [3], la contribution des dépôts serait de $20 \%$ seulement, les eaux de ruissellement étant la principale source de MES et de DCO (environ $50 \%$ ) et les eaux usées la principale source de DBO5 (55\%).

\section{XI $\square$ CONCLUSION}

Cette campagne de mesure met en évidence l'importance de la contribution du réseau d'assainissement à la pollution des RUTP. On observe une évolution des caractéristiques des eaux pluviales entre l'entrée et la sortie du réseau d'assainissement. Les concentrations des eaux de temps de pluie à l'exutoire du bassin versant sont équivalentes à celles des eaux usées et largement supérieures à celles des eaux de ruissellement. De plus, le pourcentage de pollution lié aux solides en suspension augmente entre l'amont et l'aval du bassin. D'après nos premiers résultats une grande part de la pollution rejetée par temps de pluie par les réseaux unitaires provient de l'intérieur même du réseau. Les dépôts en réseau s'avèrent être une source importante de solides mais aussi de matière organique. Cependant, ces résultats doivent être confirmés pour un nombre plus important d'événements pluvieux. Ces expérimentations ainsi que les calculs de bilan vont être poursuivis et approfondis dans l'année à venir. Une étude est en cours sur la variabilité des concentrations des eaux de ruissellement à l'échelle du bassin versant, afin d'affiner les bilans.

\section{RÉFÉRENCES}

[1] Saget A. (1994). - Base de données sur la qualité des rejets urbains de temps de pluie : distribution de la pollution rejetée, dimension des ouvrages d'interception. Thèse de doctorat, Ecole Nationale des Ponts et Chaussées, Paris, France, $333 \mathrm{p}$.

[2] Aiguier E., Chebbo G., Bertrand-Krajewsisi J.-L., Hedges P., Tyack N. (1996), - Methods for determining the settling velocity profiles of solids in storm sewage. Wat.Sci.Tech. Vol. $33 \mathrm{~N}^{\circ}$ 9, p 117-125.

[3] Cheввo G. (1992). - Solides des rejets urbains par temps de pluie : caractérisation et traitabilité. Thèse de doctorat, Ecole Nationale des Ponts et Chaussées, Paris, France, $410 \mathrm{p}+$ annexes.

[4] Krejci V., Dauber L., Novak B. and Gujer W. (1987). Contribution of different sources to pollutant loads in combined sewers. Proc. 4th Int.Conf. on Urban Storm Drainage, Lausanne, Swizerland, Aug 31-Sept. 4, 34-39.

[5] Saget A. and Chebro G. (1996). - Pollution loads of urban wet weather discharges. Proc. 7th Int.Conf. on Urban Storm Drainage, Hannover, Germany, Sept. 9-13, 61-66.

[6] Degrémont (1989). - Mémento technique de l'eau. Paris, Lavoisier : Technique et Documentation, 1459 p..

[7] Chebbo G., Mouchel. J.M., Saget A. and Gousallles M. (1995). - La pollution des rejets urbains par temps de pluie : flux, nature et impacts. T.S.M., 11, 796-806.

[8] BACHOC A. (1992). - Le transfert des solides en réseaux d'assainissement unitaires. Thèse de doctorat, Institut National Polytechnique de Toulouse, Toulouse, France, 281 p. + annexes.

[9] Gromaire-Mertz M.C., Garnaud S., Gonzalez A., Duclos Y., SaAd M., Chebbo G., Mouchel J.M., ThÉvenot D. (1997). - Le bassin versant expérimental urbain du marais à Paris. Actes du colloque « Eaux dans la Ville », 13-14 mars, Bordeaux, France. 